\title{
A New Space-Time MIMO Channel Model
}

\author{
Min Zhang, Peter J. Smith and Mansoor Shafi
}

\begin{abstract}
In this paper we develop a MIMO channel model and derive its spatial and temporal correlation properties. We present a generalized methodology to derive the spatial correlation when the angles of arrival (AoA) and angles of departure are either independent or fully correlated. Our model therefore spans the full range from well-established single ring models, where AoA and AoD are fully correlated to complex industrial channel models where they are uncorrelated. We compare our model to other MIMO channel models in terms of correlation structure and mutual information. Finally, it is shown that first order and second order approximations to the channel give rise to a single-Kronecker model and a sum-Kronecker model respectively.
\end{abstract}

Index Terms-MIMO, channel models, polarization

\section{INTRODUCTION}

The number of MIMO channel models available in the literature is rapidly growing [1]-[5] and their complexity is increasing [5], [6]. Many of these models have the now well-known Kronecker form [2]-[4] and result from the assumption of separate scattering mechanisms at the base station (BS) and the mobile station (MS). In this paper we refer to this form as a "single-Kronecker " structure to differentiate it from an alternative form we call a "sum-Kronecker" structure. Although a singleKronecker model is popular and shows good agreement with some measured data [3], [4], [7], its accuracy has been questioned [8]. Commonly used one-ring models [1], [9], [10] are by their nature "non-Kronecker", since the scattering mechanisms are linked. For these one-ring models the angle of departure (AoD) of a ray uniquely determines the angle of arrival (AoA). This observation motivates the development of a model which will bridge the gap between one-ring models, where AoD and AoA are fully correlated, and models where AoD and AoA may only be loosely related and a single-Kronecker structure is plausible. The suitability of a Kronecker structure has been studied in [11].

In this paper, we develop such a model, always bearing in mind a desire for mathematical simplicity, physical reality and ease of generation. The model builds on the model proposed by Abdi and Kaveh [1]. Two approximations are studied which give particularly simple singleKronecker and sum-Kronecker structures. We refer to a sum-Kronecker structure when the correlation matrix

Min Zhang and Peter J. Smith are with the Department of Electrical and Computer Engineering, The University of Canterbury, Private Bag 4800 Christchurch, New Zealand.

E-mail: Min Zhang: mzh22estudent.canterbury.ac.nz Peter J. Smith: p.smith@elec.canterbury.ac.nz.

Mansoor Shafi is with Telecom New Zealand, PO Box 293, Wellington, New Zealand, E-mail: Mansoor. Shafi@telecom.co.nz. can be expressed as the sum of two Kronecker products. The new model is then compared to standard one-ring models and Kronecker models. The mutual information (MI) of the MIMO link is also considered and we show the relationship between MI, orientation and the level of correlation between AoD and AoA.

The main contributions of the paper are the following:

- An extension of the one-ring model to allow for varying degrees of correlation between AoA and AoD. An approximation of this model provides a simple sum-Kronecker structure which is more general than the traditional single-Kronecker format.

- New results on the effect of non-Kronecker correlation structures on MI. We show that nonKronecker correlation does not necessarily increase the MI as previously reported [9], [10].

- New results for the spatial correlation at the BS which agree with SCM132 [5] but decay more rapidly and with less oscillation than the correlation predicted by one-ring models.

\section{FREQUENCY NONSELECTIVE RAYLEIGH CHANNEL FOR THE MIMO LINK}

Let us consider a $\left(n_{t}, n_{r}\right)$ wireless MIMO system with $n_{t}$ BS and $n_{r}$ MS omnidirectional antenna elements, shown in Fig. 1. The BS transmits signals with a narrow beamwidth $\Delta$ and the MS receives them from a large number of local scatterers surrounding the MS. The relationship between the link distance $D$ and the radius of the scatterer ring $R$ is determined by $\Delta$, that is $\tan (\Delta)=R / D$. We will assume that $D \gg R$. The MS moves with speed $v$ and the direction of motion is $\theta_{v}$. The ring of scatterers is assumed to be independent of time so that the accuracy of spatial-temporal correlations given by the model are most reliable for short periods of time $(\ll R / v)$. We do not consider line-of-sight (LOS) in the system since one of our main aims is to analyze the separability of the channel correlation structure and a LOS path fundamentally ties the MS and BS effects. For example, McNamara etc. at [12] have found the singleKronecker structure to be reasonable only under NLOS propagation.

Considering a downlink system, in our model each AoA is not associated with one specified AoD as in onering models [1], but a cluster of M subpaths (AoDs) with given power azimuth spectrum (PAS). These particular subpaths leave the $s^{\text {th }}$ array element, $B S_{s}$, impinging on the $u^{t h}$ array element, $M S_{u}$, in the direction of $\theta_{A o A}$ after being scattered by $S_{i}$ and combining. We only model a single ray at the MS which can be interpreted 


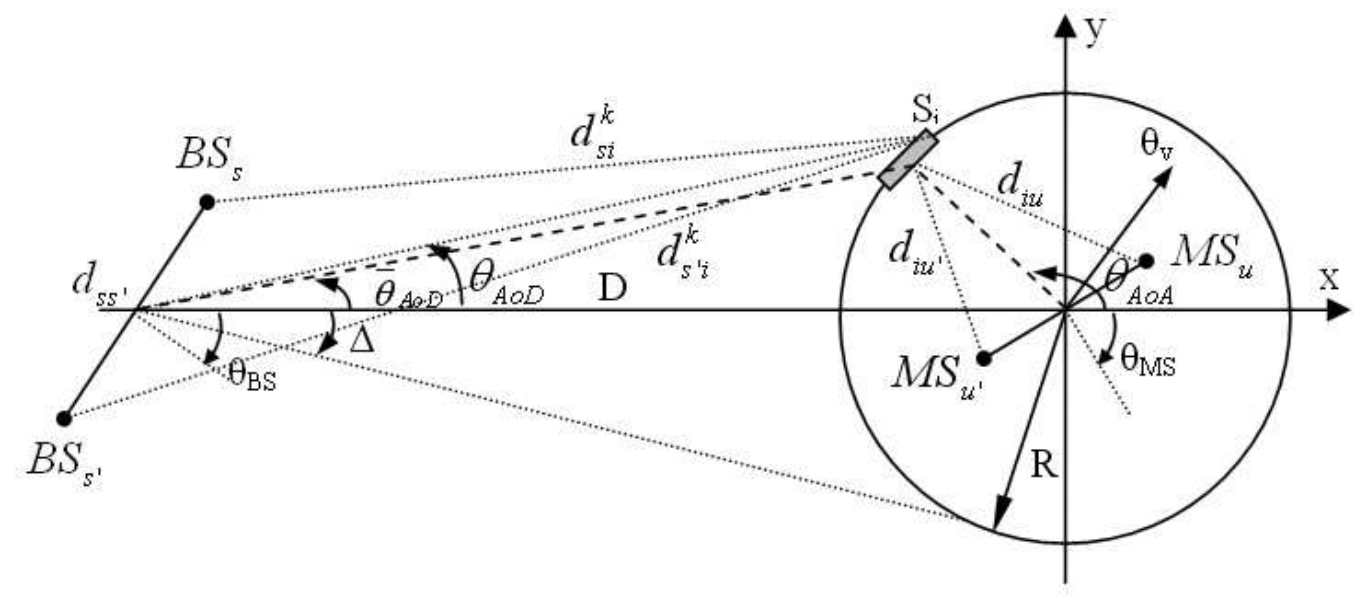

1: Co-polarized MIMO model

as a sum over subrays from scatterer $S_{i}$. A mathematical representation of this propagation model is given below using a similar derivation to that in [1]

$$
\begin{aligned}
\rho_{s u, s^{\prime} u^{\prime}} & \left(\tau, \theta_{v}\right)=\lim _{N \rightarrow \infty} \lim _{M \rightarrow \infty} \sum_{i=1}^{N} g_{i}^{2} \times \\
& \left\{\sum_{k=1}^{M}\left(g_{i}^{k}\right)^{2} \exp \left(-\frac{j 2 \pi}{\lambda}\left(d_{s i}^{k}-d_{s^{\prime} i}^{k}\right)\right)\right\} \times \\
& \exp \left(-\frac{j 2 \pi}{\lambda}\left(d_{i u}-d_{i u^{\prime}}\right)-j 2 \pi f_{D} \cos \left(\theta_{A o A}-\theta_{v}\right) \tau\right)
\end{aligned}
$$

$h_{s u}(t)=\lim _{N \rightarrow \infty} \lim _{M \rightarrow \infty} \sum_{i=1}^{N} g_{i}\left\{\sum_{k=1}^{M} g_{i}^{k} \exp \left(j \psi_{i}^{k}-\frac{j 2 \pi d_{s i}^{k}}{\lambda}\right)\right\}$

$$
\exp \left(-\frac{j 2 \pi d_{i u}}{\lambda}+j 2 \pi f_{D} \cos \left(\theta_{A o A}-\theta_{v}\right) t\right)
$$

where $h_{s u}(t)$ is the channel coefficient and $\mathbf{H}(t)=$ $\left(h_{s u}(t)\right)$ is the channel matrix. Other parameters in (1) are defined below. $\mathrm{N}$ is the number of scatterers, $g_{i}$ is the wave amplitude of the $i$ th path where $\sum_{i=1}^{N}\left\|g_{i}\right\|^{2}=1$ as $N \rightarrow \infty$. Each path is the sum of $\mathrm{M}$ subpaths; $g_{i}^{k}$ is the wave amplitude of the $k$ th subpath of the $i$ th path where $\sum_{k=1}^{M}\left\|g_{i}^{k}\right\|^{2}=1$ as $M \rightarrow \infty$. $\psi_{i}^{k}$ are the phase shifts introduced by the scatterers and are assumed to be independent and identically distributed (iid) uniform variables over $[0,2 \pi) . d_{s i}^{k}$ and $d_{i u}$ are the distances from $B S_{s}$ to $S_{i}$ and from $S_{i}$ to $M S_{u}$ respectively. Finally, $f_{D}=v / \lambda$ is the maximum Doppler shift. As in [1], the summation over many paths leads to a Gaussian channel coefficient and so we have a Rayleigh channel.

For a fixed direction of motion, $\theta_{v}$, we define the temporal-spatial correlation function for the channel coefficients as $\rho_{s u, s^{\prime} u^{\prime}}\left(\tau, \theta_{v}\right)=\mathbb{E}\left[h_{s u}(t) h_{s^{\prime} u^{\prime}}^{*}(t+\tau)\right]$. We note that $\mathbb{E}\left[\exp \left(j \psi_{i}^{k}-j \psi_{i^{\prime}}^{k^{\prime}}\right)\right]=1$ for $i=i^{\prime}$ and $k=k^{\prime}$ and is zero otherwise. Therefore, $\rho_{s u, s^{\prime} u^{\prime}}\left(\tau, \theta_{v}\right)$ can be written as
Now we use the von-Mises PAS at both ends following [1] [13]. The von Mises PAS is given by

$$
f(\theta)=\frac{\exp [\kappa \cos (\theta-\bar{\theta})]}{2 \pi I_{0}(\kappa)}, \quad \theta \in[-\pi, \pi)
$$

where $I_{(.)}$is a modified Bessel function and $\kappa$ controls the width of angle spread (AS). In fact, $\kappa$ is inversely proportional to the AS, with $\kappa=0$ giving a uniform spread of angles over $360^{\circ}$ and $\kappa=\infty$ giving a ray at the single angle $\bar{\theta}$. In this paper we use $\kappa_{M S}=0.5$ at the MS which falls in the range of values used in [13]. At the BS end, we use $\kappa_{B S}=100$ and $\kappa_{B S}=$ 500. Simulation of SCM132 [5] for the suburban macro scenario gives a very similar value to $\kappa_{B S}=100$. These values correspond to a $95 \%$ AS of about $22^{\circ}$ (for $\kappa_{B S}=$ $100)$ and $10^{\circ}$ (for $\kappa_{B S}=500$ ).

$\bar{\theta}$ is the mean direction and this direction has maximum power. Hence $\bar{\theta}_{A o A}$ represents the direction of the strongest incoming wave seen by the user. Moreover, $\bar{\theta}_{A o D}$ is the direction of the strongest incoming wave seen from a specified scatterer, which can be determined by $\theta_{A o A}$. As $D \gg R$ and $\Delta=R / D$ is small, $\bar{\theta}_{A o D}$ can be simplified as below [1]

$$
\begin{aligned}
\bar{\theta}_{A o D} & \approx \sin \left(\bar{\theta}_{A o D}\right) \approx R / D \sin \left(\theta_{A o A}\right) \\
& =\Delta \sin \left(\theta_{A o A}\right)
\end{aligned}
$$


Hence the power of every path or subpath is given by

$$
\begin{aligned}
\left(g_{i}\right)^{2} & =\frac{\exp \left[\kappa_{M S} \cos \left(\theta_{A o A}-\bar{\theta}_{A o A}\right)\right]}{2 \pi I_{0}\left(\kappa_{M S}\right)} d \theta_{A o A} \\
\left(g_{i}^{k}\right)^{2} & =\frac{\exp \left[\kappa_{B S} \cos \left(\theta_{A o D}-\Delta \sin \left(\theta_{A o A}\right)\right)\right]}{2 \pi I_{0}\left(\kappa_{B S}\right)} d \theta_{A o D}
\end{aligned}
$$

The larger the value of $\kappa_{B S}$, the narrower the cluster of subpaths will be and the power of these subpaths will concentrate around the mean direction. As $\kappa_{B S} \rightarrow \infty$, they will converge into one ray and make AoA and AoD fully correlated. Moreover, under the sensible physical scenario where $D \gg d_{s s^{\prime}}$ and $R \gg d_{u u^{\prime}}$ [14], (2) can be simplified further using

$$
\begin{aligned}
& \frac{2 \pi}{\lambda}\left(d_{s i}^{k}-d_{s^{\prime} i}^{k}\right) \approx D_{s s^{\prime}} \sin \left(\theta_{A o D}-\theta_{B S}\right) \\
& \frac{2 \pi}{\lambda}\left(d_{i u}-d_{i u^{\prime}}\right) \approx D_{u u^{\prime}} \sin \left(\theta_{A o A}-\theta_{M S}\right)
\end{aligned}
$$

where $D_{s s^{\prime}}=2 \pi d_{s s^{\prime}} / \lambda$ and $D_{u u^{\prime}}=2 \pi d_{u u^{\prime}} / \lambda$ are the distances between the antenna elements in wavelengths. Substituting equations (5) and (6) into (2) and defining $D_{t}=2 \pi f_{D} \tau$, the correlation averaged over AoA and AoD can be expressed as

$$
\begin{aligned}
& \rho_{s u, s^{\prime} u^{\prime}}\left(\tau, \theta_{v}\right) \approx \frac{1}{2 \pi I_{0}\left(\kappa_{B S}\right)} \frac{1}{2 \pi I_{0}\left(\kappa_{M S}\right)} \int_{-\pi}^{\pi} \int_{-\pi}^{\pi} \\
& \exp \left\{-j D_{s s^{\prime}} \sin \left(\theta_{A o D}-\theta_{B S}\right)\right. \\
& \left.\left.\quad+\kappa_{B S} \cos \left(\theta_{A o D}-\Delta \sin \left(\theta_{A o A}\right)\right)\right)\right\} \\
& \exp \left\{-j D_{u u^{\prime}} \sin \left(\theta_{A o A}-\theta_{M S}\right)+\kappa_{M S} \cos \left(\theta_{A o A}-\bar{\theta}_{A o A}\right)\right\} \\
& \exp \left\{-j D_{t} \cos \left(\theta_{A o A}-\theta_{v}\right)\right\} d\left(\theta_{A o A}\right) d\left(\theta_{A o D}\right)
\end{aligned}
$$

Since $\Delta$ is small, we are able to use the approximations, $\cos \left(\Delta \sin \left(\theta_{A o A}\right)\right) \approx 1$ and $\sin \left(\Delta \sin \left(\theta_{A o A}\right)\right) \approx$ $\Delta \sin \left(\theta_{A o A}\right)$, in (7). This gives

$\rho_{s u, s^{\prime} u^{\prime}}\left(\tau, \theta_{v}\right) \approx \frac{1}{2 \pi I_{0}\left(\kappa_{B S}\right)} \frac{1}{2 \pi I_{0}\left(\kappa_{M S}\right)} \int_{-\pi}^{\pi} \int_{-\pi}^{\pi}$

$\left.\exp \left\{-j D_{s s^{\prime}} \sin \left(\theta_{A o D}-\theta_{B S}\right)+\kappa_{B S} \cos \left(\theta_{A o D}\right)\right)\right\}$

$\exp \left\{-j D_{u u^{\prime}} \sin \left(\theta_{A o A}-\theta_{M S}\right)+\kappa_{M S} \cos \left(\theta_{A o A}-\bar{\theta}_{A o A}\right)\right\}$ and the first-order approximation is

$\exp \left\{\kappa_{B S} \Delta \sin \left(\theta_{A o A}\right) \sin \left(\theta_{A o D}\right)\right\}$

$\exp \left\{-j D_{t} \cos \left(\theta_{A o A}-\theta_{v}\right)\right\} d\left(\theta_{A o A}\right) d\left(\theta_{A o D}\right)$

We assume that $\theta_{v}$ is an independent variable with uniform distribution over $[0,2 \pi)$. This leads to the well known Bessel function term $J_{0}\left(D_{t}\right)$ after taking expectation over $\theta_{v}$. Therefore the temporal-spatial correlation coefficient after averaging over $\theta_{v}$ is given by:

$$
\begin{aligned}
& \rho_{s u, s^{\prime} u^{\prime}}(\tau) \approx J_{0}\left(D_{t}\right) \frac{1}{2 \pi I_{0}\left(\kappa_{B S}\right)} \frac{1}{2 \pi I_{0}\left(\kappa_{M S}\right)} \int_{-\pi}^{\pi} \int_{-\pi}^{\pi} \\
& \left.\exp \left\{-j D_{s s^{\prime}} \sin \left(\theta_{A o D}-\theta_{B S}\right)+\kappa_{B S} \cos \left(\theta_{A o D}\right)\right)\right\} \\
& \exp \left\{-j D_{u u^{\prime}} \sin \left(\theta_{A o A}-\theta_{M S}\right)+\kappa_{M S} \cos \left(\theta_{A o A}-\bar{\theta}_{A o A}\right)\right\} \\
& \exp \left\{\kappa_{B S} \Delta \sin \left(\theta_{A o A}\right) \sin \left(\theta_{A o D}\right)\right\} \\
& d\left(\theta_{A o A}\right) d\left(\theta_{A o D}\right)
\end{aligned}
$$

Note that (9) has 4 types of terms. The first term, $J_{0}\left(D_{t}\right)$, represents temporal correlation. The second term is the exponential containing BS parameters which re-presents spatial correlation at the BS. Similarly, the third term is the the exponential containing MS parameters which represents spatial correlation at the MS. The last term, $\exp \left\{\kappa_{B S} \Delta \sin \left(\theta_{A o A}\right) \sin \left(\theta_{A o D}\right)\right\}$, represents the interaction between AoA and AoD and its effect on the correlation. This is the factor which affects the separability of the correlation structure. Equation (9) gives the full correlation structure for the model. Computation of (9) requires double numerical integration and for this reason we prefer to investigate approximations based on the series expansion $\exp \left\{\kappa_{B S} \Delta \sin \left(\theta_{A o A}\right) \sin \left(\theta_{A o D}\right)\right\} \approx 1+$ $\kappa_{B S} \Delta \sin \left(\theta_{A o A}\right) \sin \left(\theta_{A o D}\right)$. We define the zeroth-order and first-order approximations as resulting from taking 1 or 2 terms in the above series expansion respectively. With these approximations the correlation function in (9) can be simplified considerably using the standard results [3.937,p488, [15]]

$$
\begin{aligned}
& \int_{-\pi}^{\pi} \exp (p \cos x+q \sin x) d x \\
& \quad=2 \pi I_{0}\left(\sqrt{p^{2}+q^{2}}\right) \\
& \int_{-\pi}^{\pi} \exp (p \cos x+q \sin x) \sin x d x \\
& \quad=2 \pi \frac{-q}{\sqrt{p^{2}+q^{2}}} I_{1}\left(\sqrt{p^{2}+q^{2}}\right)
\end{aligned}
$$

Using (10), the zeroth-order approximation of (9) is

$$
\rho_{s u, s^{\prime} u^{\prime}}(\tau) \approx J_{0}(\tau) \frac{I_{0}\left(\sqrt{p_{B S}^{2}+q_{B S}^{2}}\right)}{I_{0}\left(\kappa_{B S}\right)} \frac{I_{0}\left(\sqrt{p_{M S}^{2}+q_{M S}^{2}}\right)}{I_{0}\left(\kappa_{M S}\right)}
$$

$$
\begin{aligned}
\rho_{s u, s^{\prime} u^{\prime}}(\tau) \approx J_{0}(\tau) \frac{I_{0}\left(\sqrt{p_{B S}^{2}+q_{B S}^{2}}\right)}{I_{0}\left(\kappa_{B S}\right)} \frac{I_{0}\left(\sqrt{p_{M S}^{2}+q_{M S}^{2}}\right)}{I_{0}\left(\kappa_{M S}\right)} \\
+J_{0}(\tau)\left\{\frac{-\Delta \kappa_{B S} q_{B S}}{\sqrt{p_{B S}^{2}+q_{B S}^{2}}} \frac{I_{1}\left(\sqrt{p_{B S}^{2}+q_{B S}^{2}}\right)}{I_{0}\left(\kappa_{B S}\right)}\right\} \\
\quad \times\left\{\frac{-q_{M S}}{\sqrt{p_{M S}^{2}+q_{M S}^{2}}} \frac{I_{1}\left(\sqrt{p_{M S}^{2}+q_{M S}^{2}}\right)}{I_{0}\left(\kappa_{M S}\right)}\right\}
\end{aligned}
$$


where

$$
\begin{aligned}
& p_{B S}=\kappa_{B S}+j D_{s s^{\prime}} \sin \left(\theta_{B S}\right) \\
& q_{B S}=-j D_{s s^{\prime}} \cos \left(\theta_{B S}\right) \\
& p_{M S}=\kappa_{M S} \cos \left(\bar{\theta}_{A o A}\right)+j D_{u u^{\prime}} \sin \left(\theta_{M S}\right) \\
& q_{M S}=\kappa_{M S} \sin \left(\bar{\theta}_{A o A}\right)-j D_{u u^{\prime}} \cos \left(\theta_{M S}\right)
\end{aligned}
$$

For the zeroth order approximation, (11) gives rise to a single-Kronecker result for the channel correlation matrix, $\mathbf{R}_{H}(\tau)=\mathbb{E}\left(\operatorname{vec}(\mathbf{H}(t)) \operatorname{vec}(\mathbf{H}(t))^{\dagger}\right)$ as below

$$
\mathbf{R}_{H}(\tau)=J_{0}(\tau)\left(\mathbf{R}_{B S}^{0} \bigotimes \mathbf{R}_{M S}^{0}\right)
$$

For the first order approximation a sum-Kronecker form is given:

$$
\mathbf{R}_{H}(\tau)=J_{0}(\tau)\left(\mathbf{R}_{B S}^{0} \bigotimes \mathbf{R}_{M S}^{0}+\mathbf{R}_{B S}^{1} \bigotimes \mathbf{R}_{M S}^{1}\right)
$$

In (13) and (14), $\bigotimes$ is defined as the Kronecker product and the correlation matrices $\mathbf{R}_{B S}^{0}, \mathbf{R}_{M S}^{0}, \mathbf{R}_{B S}^{1}, \mathbf{R}_{M S}^{1}$ are Hermitian matrices.

It is interesting that the new model collapses to a single-Kronecker model when the zero order approximation is used and the first order approximation retains the correlation between AoD and AoA via a second Kronecker term. Hence the model encapsulates the AoD-AoA correlation with a logical extension of the single-Kronecker to a sum-Kronecker form and retains a similar concise mathematical structure.

\section{Simulation Results}

Here we simulate an $\left(n_{t}, n_{r}\right)$ MIMO system. Unless otherwise stated, we assume that $n_{t}=n_{r}=8, \Delta=2^{\circ}$, $\bar{\theta}_{A o A}=0, \theta_{B S}=\theta_{M S}=0, \kappa_{B S}=500, \kappa_{M S}=0.5$, $d_{s s^{\prime}}=\lambda, d_{u u^{\prime}}=0.5 \lambda, S N R=20 d B$.

\section{A. Spatial Correlation}

Firstly we compare the new MIMO model (12) with the one-ring model in [1]. Based on our new model, we have

$$
\begin{aligned}
& \rho_{11,21}(0)=\frac{I_{0}\left(\sqrt{\kappa_{B S}^{2}-D_{s s^{\prime}}^{2}}\right)}{I_{0}\left(\kappa_{B S}\right)} \\
& \rho_{11,12}(0)=\frac{I_{0}\left(\sqrt{\kappa_{M S}^{2}-D_{u u^{\prime}}^{2}}\right)}{I_{0}\left(\kappa_{M S}\right)}
\end{aligned}
$$

whereas [1] gives

$$
\begin{aligned}
& \rho_{11,21}(0)=\frac{I_{0}\left(\sqrt{\kappa_{M S}^{2}-D_{s s^{\prime}}^{2} \Delta^{2}}\right)}{I_{0}\left(\kappa_{M S}\right)} \\
& \rho_{11,12}(0)=\frac{I_{0}\left(\sqrt{\kappa_{M S}^{2}-D_{u u^{\prime}}^{2}}\right)}{I_{0}\left(\kappa_{M S}\right)}
\end{aligned}
$$

We can see that the correlation coefficients are the same at the MS but not at the BS. Results are shown in Fig. 2 and Fig. 3 for different antenna spacings and $\kappa_{B S}$

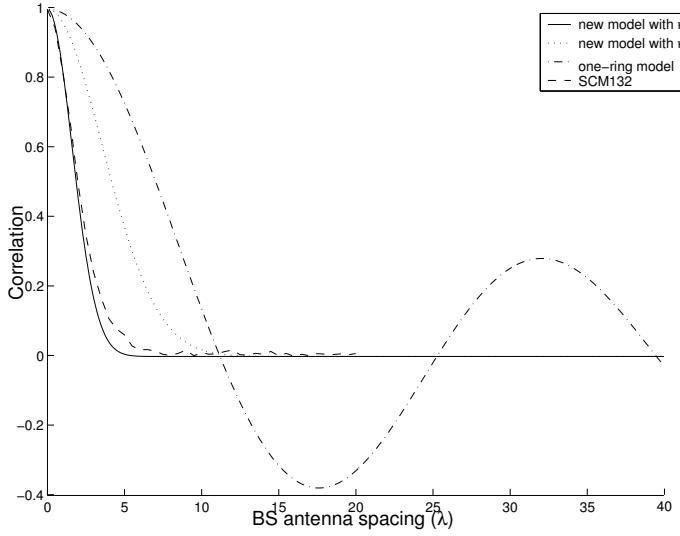

2: The BS correlation $\rho_{11,21}(0)$

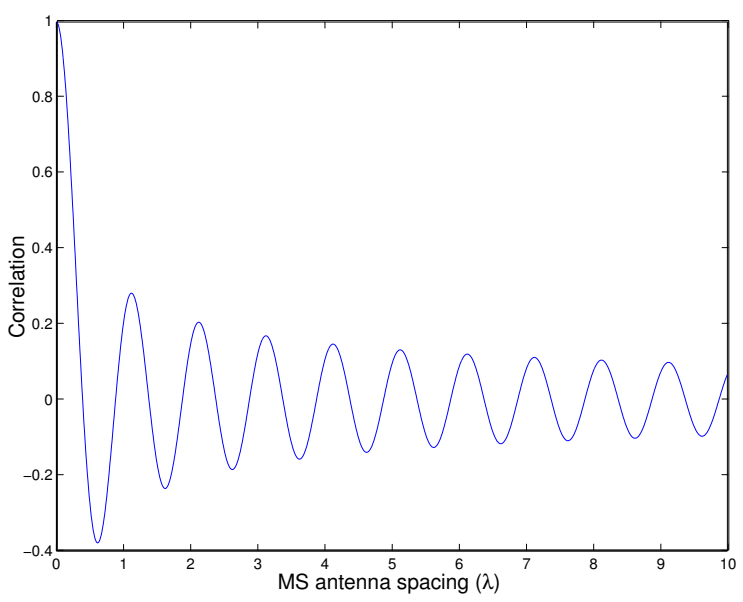

3: The MS correlation $\rho_{11,12}(0)$

values. The one-ring model shows large oscillating correlations at the BS even for large antenna spacings. The new model suggests that the correlation will decrease roughly exponentially, the speed of decay is related to the value of $\kappa_{B S}$ and the pronounced oscillations are absent. This type of result agrees with simulations of the SCM132 model [5] and measured data [16] (see Fig. 2).

\section{B. Approximation Order}

In order to show that the zeroth-order and firstorder approximations are reasonable, we compare the full correlation (7) (no approximation) with (11) (zeroth-order approximation) and (12) (first-order approximation) in a co-polarized $(4,4)$ MIMO system. The calculation of (7) uses double adaptive Simpson quadrature and the absolute error tolerance is set to be $1 E-8$. The relative difference between any two correlation matrices $\mathbf{R}_{1}$ and $\mathbf{R}_{2}$ is defined as $100 *$ $\left\|\mathbf{R}_{1}-\mathbf{R}_{2}\right\| /\left\|\mathbf{R}_{1}\right\|$ where $\|$.$\| is the Frobenius norm.$ The results are shown in Table I. We can see that the first-order approximation performs better than the zerothorder approximation by $0-7 \%$. It also shows that the larger the value of $\kappa_{B S}$, the larger the errors will be. 


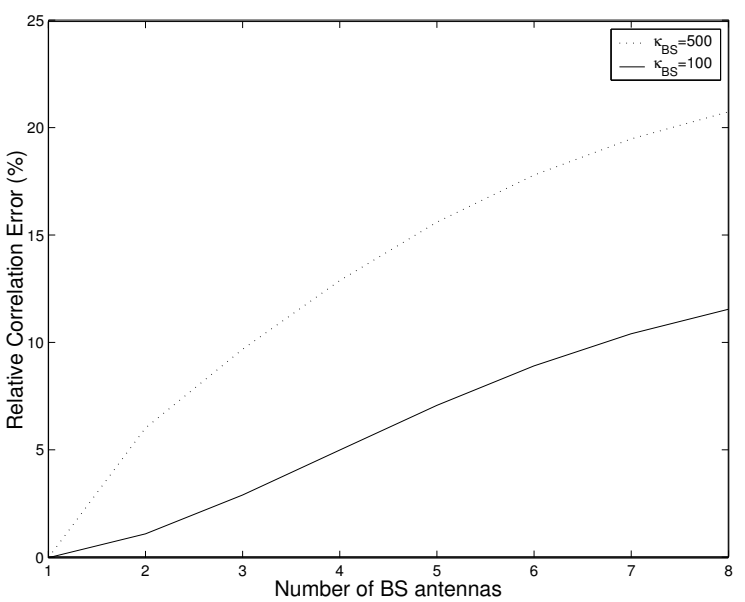

4: Relative correlation error vs the number of BS antennas for a $\left(n_{t}, 4\right)$ system

Moreover, from Fig. 4, increasing the number of antenna elements will also increase the error, which agrees with [4]. Therefore, a single-Kronecker structure is more suited for MIMO systems with small number of antenna elements and in all cases, a first-order approximation will improve the accuracy of the model.

\section{Mutual Information of the MIMO System}

If we use a single-Kronecker structure in the copolarized model, the channel matrix is given by

$$
\mathbf{H}(t)=\left(\mathbf{R}_{M S}^{0}\right)^{\frac{1}{2}} \mathbf{U}_{0}(t)\left(\mathbf{R}_{B S}^{0}\right)^{\frac{1}{2} T}
$$

where $\mathbf{R}^{\frac{1}{2}}$ is the matrix square root of a Hermitian matrix $\mathbf{R}$, superscript $T$ denotes transpose and $\mathbf{U}_{(\text {.) }}$ is an iid Gaussian channel matrix generated by the Jakes Model with zero mean and unit magnitude variance.

This standard generation method works for the zeroth order approximation as $\mathbf{R}_{(.)}^{0}$ is Hermitian non-negative definite. For the first order approximation a complication arises since $\mathbf{R}_{(.)}^{1}$ can have negative eigenvalues. Hence we use an equivalent structure which avoids this problem. Using this approach the channel matrix for the first order approximation is

$$
\begin{aligned}
\mathbf{H}(t)= & \left(\frac{\mathbf{R}_{M S}^{0}-\mathbf{R}_{M S}^{1}}{\sqrt{2}}\right)^{\frac{1}{2}} \mathbf{U}_{0}(t)\left(\frac{\mathbf{R}_{B S}^{0}-\mathbf{R}_{B S}^{1}}{\sqrt{2}}\right)^{\frac{1}{2} T} \\
& +\left(\frac{\mathbf{R}_{M S}^{0}+\mathbf{R}_{M S}^{1}}{\sqrt{2}}\right)^{\frac{1}{2}} \mathbf{U}_{1}(t)\left(\frac{\mathbf{R}_{B S}^{0}+\mathbf{R}_{B S}^{1}}{\sqrt{2}}\right)^{\frac{1}{2} T}
\end{aligned}
$$

It can be shown that the matrices of the form $\mathbf{R}_{(.)}^{0} \pm$ $\mathbf{R}_{(.)}^{1}$ may still have negative eigenvalues but they are negligible compared to the other eigenvalues. Hence we can remove them and make the correlation matrices nonnegative definite with almost no loss in accuracy.

The mutual information (MI) of MIMO system is denoted by $\mathcal{I}$ and is given by
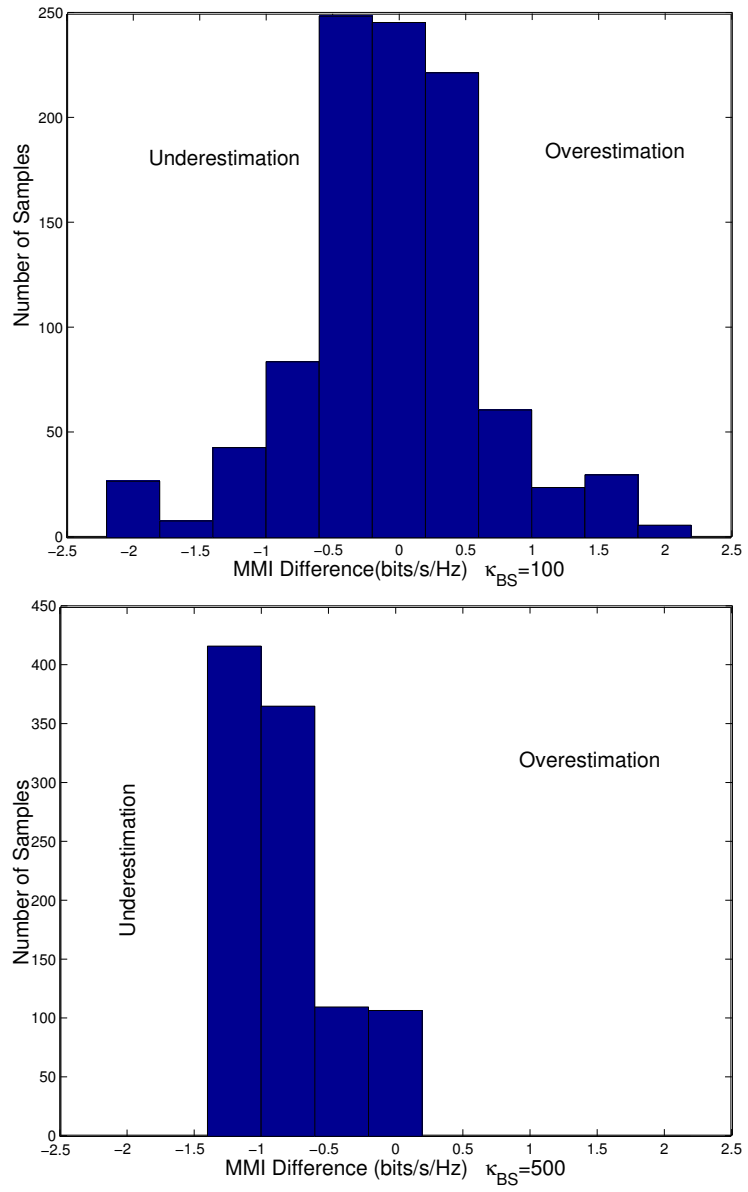

5: MMI difference between the zeroth and first order approximations

$$
\mathcal{I}=\log _{2}\left[\operatorname{det}\left(\mathbf{I}+\frac{S N R}{n_{B S}} \mathbf{H} \mathbf{H}^{\dagger}\right)\right]
$$

where superscript $\dagger$ denotes the transpose conjugate. Fig. 5 shows the difference in the mean mutual information (MMI) given by the single-Kronecker and sum-Kronecker structure for a MIMO system when we randomly choose the orientations of the BS and the MS. Specifically, Fig. 5 plots the MMI difference $\mathbb{E}(\mathcal{I}($ zeroth order $))-\mathbb{E}(\mathcal{I}$ (first order $))$ and results are obtained by simulation. We can see that the singleKronecker structure normally underestimates the channel MMI compared to the sum-Kronecker structure when the correlation of AoA and AoD is large. However, when the correlation is small, this structure may overestimate or underestimate the MMI with almost equal probability. The beneficial impact of non-Kronecker channel correlation on MIMO MMI has been reported in [9], [10] but this may be too optimistic. If AoD and AoA are only loosely related,the additional channel correlation may be beneficial or detrimental depending on the orientation of the BS and MS. Therefore, the single-Kronecker model tends to systematically underestimate the MMI 


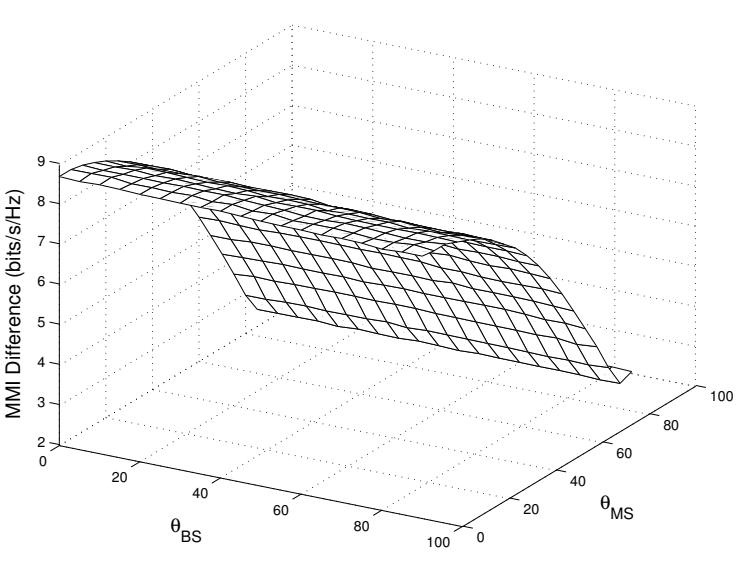

6: MMI difference vs antenna array orientation

only when the correlation of AoD and AoA is strong.

Fig. 6 shows how MMI varies for small and large correlations of AoA and AoD in a co-polarized MIMO system using the sum-Kronecker model. The MMI difference plotted is $\mathbb{E}\left(\mathcal{I}\left(\kappa_{B S}=100\right)\right)-\mathbb{E}\left(\mathcal{I}\left(\kappa_{B S}=\right.\right.$ $500))$. Fig. 6 shows that the smaller the correlation, the larger the channel MMI will be. This improvement is largest when the MS is facing the BS and smallest when it is at $90^{\circ}$.

\section{CONCLUSION}

In this paper we have derived an extension to the popular one-ring model in [1]. The new model allows for varying degrees of correlation between the AoD and AoA of the departing and arriving rays. Approximations to the new model give rise to a zeroth order single-Kronecker approximation and a first order sumKronecker approximation. Hence the correlation structure remains mathematically concise for both approximations and suggests that the sum-Kronecker model may be a sensible general model in non-Kronecker scenarios. Spatial correlations at the BS derived from the new model are substantially different to those in [1] but agree with those in [5]. In particular, the spatial correlation decays smoothly with antenna spacing and is negligible at high spacings. Finally, using the new model we show that non-Kronecker correlation does not necessarily increase the MI as previously reported. Our results demonstrate that MI can be increased or decreased depending on the orientation and the correlation between AoA and AoD.

\section{REFERENCES}

[1] A. Abdi and M.Kaveh, "A space-time correlation model for multielement antenna systems in mobile fading channels", IEEE J. on Selected Areas in Comm., Vol. 20, No. 3, pp. 550 - 560, April 2002

[2] D. Shiu, G.J. Foschini, M. J. Gans and J. M. Kahn, "Fading correlation and its effect on the capacity of multi-element antenna systems", IEEE Trans. on Comm., Vol. 48, No. 3, pp. 502 - 513 , March 2000

\begin{tabular}{|r|r|r|r|r|r|}
\hline \multicolumn{2}{|c|}{} & \multicolumn{2}{c|}{$\kappa_{B S}=100$} & \multicolumn{2}{c|}{$\kappa_{B S}=500$} \\
\hline$\theta_{B S}$ & $\theta_{M S}$ & Zeroth & First & Zeroth & First \\
\hline 0 & 0 & 8.41 & 2.50 & 12.92 & 5.02 \\
\hline$\pi / 6$ & 0 & 8.03 & 2.26 & 11.36 & 3.96 \\
\hline$\pi / 3$ & 0 & 5.86 & 1.17 & 6.73 & 1.48 \\
\hline 0 & $\pi / 6$ & 7.50 & 2.11 & 11.45 & 4.23 \\
\hline$\pi / 6$ & $\pi / 6$ & 7.16 & 1.90 & 10.07 & 3.33 \\
\hline$\pi / 3$ & $\pi / 6$ & 5.22 & 0.98 & 5.97 & 1.25 \\
\hline 0 & $\pi / 3$ & 4.71 & 1.64 & 7.28 & 3.30 \\
\hline$\pi / 6$ & $\pi / 3$ & 4.49 & 1.48 & 6.37 & 2.59 \\
\hline$\pi / 3$ & $\pi / 3$ & 3.25 & 0.76 & 3.72 & 0.97 \\
\hline$\pi / 4$ & $\pi / 4$ & 5.52 & 1.34 & 7.04 & 2.00 \\
\hline$\pi / 2$ & $\pi / 2$ & 0.26 & 0.26 & 0.27 & 0.27 \\
\hline
\end{tabular}

I: Relative Errors of the approximate correlation structures

[3] D. Chizhik, J. Ling, P.W. Wolniansky, R.A. Valenzuela, N. Costa and K. Huber, "Multiple-input-multiple-output measurements and modeling in Manhattan", IEEE J. on Selected Areas in Comm., Vol. 21, No. 3, pp. 321 - 331, April 2003

[4] Kai Yu, M. Bengtsson, B. Ottersten, D. McNamara, P. Karlsson, and $\mathrm{M}$. Beach "Modeling of wide-band MIMO radio channels based on NLOS indoor measurements" IEEE Trans. on Vehicular Tech., Vol. 53, No. 3, May 2004

[5] 3GPP and 3GPP2, "SCM-132: Spatial Channel Model Text description", April 2003

[6] P. J. Smith and M. Shafi, "The impact of complexity in MIMO channel models", 2004 IEEE International Conference on Communications, Vol. 5, pp. 2924 - 2928, 20-24 June 2004

[7] Kai Yu, M. Bengtsson, B. Ottersten, D. McNamara, P. Karlsson and M. Beach, "Second order statistics of NLOS indoor MIMO channels based on $5.2 \mathrm{GHz}$ measurements", IEEE Global Telecomm. Conference 2001, Vol. 1, pp. 156 - 160, 25-29 Nov. 2001

[8] H. Özcelik, M. Herdin, W. Weichselberger, J. Wallace and E. Bonek, 'Deficiencies of 'Kronecker' MIMO radio channel model", Electronics Letters, Vol. 39, No. 16, pp. 1209 - 1210, Aug. 2003

[9] C. Oestges and A.J. Paulraj, "Beneficial impact of channel correlations on MIMO capacity", Electonics Letters, Vol. 40, No. 10, pp. 606-608, 13th May, 2004

[10] C. Oestges, B. Clerckx, D. Vanhoenacker-Janvier and A. J. Paulraj, "Impact of 'diagonal' correlations on MIMO capacity: application to geometrical scattering models", 58th IEEE Vehic. Tech. Conf., Vol. 1, pp 394-398, 6-9 Oct. 2003

[11] T. S. Pollock, "Correlation modelling in MIMO systems: When can we Kronecker?", 5th Australian Communication Theory Workshop, pp 149-153, Feb. 2004

[12] D.P. McNamara, M.A. Beach and P.N. Fletcher, "Spatial correlation in indoor MIMO channels" Personal, Indoor and Mobile Radio Comm. , 2002 Vol. 1, pp. 290-294, 15-18 Sept. 2002

[13] Ali Abdi, J. A. Barger and M. Kaveh, "A parametric model for the distribution of the angle of arrival and the associated correlation function and power spectrum at the mobile station" IEEE Trans. on Vehicular Technology, Vol. 51, No. 3, pp. 425434, May 2002

[14] A. Giorgetti, M. Chiani, M. Shafi and P. J. Smith, "MIMO capacity, level crossing rates and fades: The impact of spatial/temporal channel correlation", Journal of Communications and Networks, Vol. 5, No.2, pp. 104-115, Jun. 2003

[15] I.S. Gradshteyn and I.M. Ryzhik, Table of Integral, Series, and Products, corrected and enlarged ed., A. Jeffery, Academic Press, 1980'

[16] William C. Y. Lee, "Mobile radio signal correlation versus antenna height and spacing", IEEE Trans. on Vehicular Technology, Vol 25, No. 4, pp 290-294, Aug. 1977 